\title{
A KIND OF DISTRIBUTED LASER POSITION LOCATION SYSTEM BASED ON TDOF TECHNOLOGY
}

\author{
Wenjiang $\mathrm{Li}^{1, \text { a }}$, Jian $\mathrm{Fu}^{2, \mathrm{~b}}$ \\ ${ }^{1}$ Zhejiang University, Hangzhou, Zhejiang, 310058, China \\ ${ }^{2}$ Zhejiang University, Hangzhou, Zhejiang, 310058, China \\ e289084741@qq.com, bjianfu.zju@qq.com
}

Keywords: Lase; TDOF; Positioning Algorithms; Kalman Filter.

Abstract. In the era of intelligent robots and VR technology, High-precision position technology of indoor space is increasingly important, a variety of ways of high-precision position technology to solve different practical scene problems. Based on the laser TDOF principle, this paper presents a new positioning method, its high accuracy and controllable cost show a bright future.

In this paper, we will analyze the systematic simulation, data analysis, the solution algorithm and the related trajectory processing algorithm. Two kinds of algorithms will be introduced to detect the position of the signal; the Kalman filter algorithm under predictable trajectory and the trajectory smoothing model under different motion states.

\section{Introduction}

With outdoor positioning navigation technology called GPS (Global Positioning System) increasingly matures, China has launched its own domestic satellite positioning system Beiou satellite positioning system as well. But the satellite positioning signal can't penetrate the enclosed area, so the indoor positioning signal attenuation can't use satellite positioning system.

Therefore, indoor positioning is filled with various kinds of positioning technologies, such as Bluetooth positioning, INS positioning, WIFI positioning, RFID positioning, UWB positioning, ultrasonic positioning, ZigBee positioning, geomagnetic position, Computer vision and other positioning methods.

With the popularization of VR, the corresponding positioning accuracy meet the requirement of the millimeter level. The HTC Vive Lighthouse indoor positioning technology has been applied in to the commercial computer game market, the technology belongs to laser positioning technology; The positioning technology that Oculus Rift and Sony PlayStation VR are used belongs to the optical positioning technology. Oculus Rift uses infrared active optical technology. PlayStation VR contains a visible optical active optical technology.

The technology of Lighthouse position from HTC Vive can't support a large space. According to this problem, this paper based on TDOF laser ranging technology explored a distributed measurement system, which will improve the accuracy of the position and detection of trajectory reduction through several relevant algorithms. The advantage of this system is that it has small computational complexity and it can support several units to be measured.

\section{System Model.}

In order to explain the measurement method, a simple measurement model is established, as shown in Figure 1: 


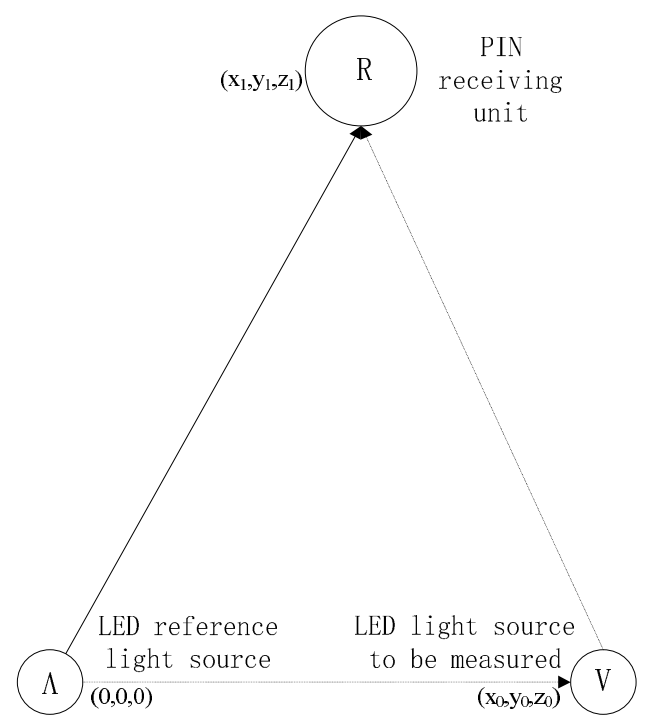

Figure 1. The basic measurement model

Infrared LED reference light source A launch a light pulse signal through a all-phase lens. Then the LED source $\mathrm{V}$ will emit a light pulse signal after a fixed delay. The fixed delay is set to $\tau_{\mathrm{s}}$. When the LED reference light pulse signal reaches to the PIN/APD photoelectric receiving unit, the rising edge triggers the high-speed pulse timer to start timing. When the light pulse signal from the LED source to be measured (V) reaches the PIN/APD photoelectric receiving unit. This rising edge triggers the high-speed pulse timer to stop timing. At the same time, the time difference of this high-speed pulse timer will be recorded as $\tau_{1}$.

If there are $\mathrm{n}$ PIN / APD photoelectric receiving units and there will be $\mathrm{n}$ time differences $\tau_{1}, \tau_{2}, \ldots$, $\tau_{\mathrm{n}}$ corresponding to the units can be obtained. LED light source to be measured can be obtained by reference to pre-set the exact coordinates of the position $(0,0,0)$, and at the same time the position of the PIN/APD photoelectric receiving units is known. And its corresponding coordinates are: $\left(\mathrm{x}_{1}, \mathrm{y}_{1}, \mathrm{z}_{1}\right)$, $\left(\mathrm{x}_{2}, \mathrm{y}_{2}, \mathrm{z}_{2}\right),\left(\mathrm{x}_{3}, \mathrm{y}_{3}, \mathrm{z}_{3}\right)$.

Assuming the coordinates of the light source to be measured is $\left(\mathrm{x}_{0}, \mathrm{y}_{0}, \mathrm{z}_{0}\right)$. This information can be estimated according to the exact position of the LED light source under test. First of all according to the coordinates of LED reference light source $(0,0,0)$ and PIN/APD photoelectric receiving unit one $\left(\mathrm{x}_{1}, \mathrm{y}_{1}, \mathrm{z}_{1}\right)$, the distance between them can be calculated:

$$
L_{1}=\sqrt{x_{1}^{2}+y_{1}^{2}+z_{1}^{2}}
$$

Then the corresponding distance for the nth photoelectric receiving unit:

$$
L_{n}=\sqrt{x_{n}^{2}+y_{n}^{2}+z_{n}^{2}}
$$

we can obtain the distance from nth receiver to the LED light source to be measured is:

$$
S_{n}=L_{n}+c\left(\tau_{1}-\tau_{s}\right)
$$

After completing the single point measurement function, If the system wants to complete the more complex indoor positioning function, it is necessary to achieve simultaneous multi-point measurement. Therefore, on the basis of single-point measurement, a set of multi-point simultaneous measurement system is designed. The time division multiplexing system is showed in Figure 2: 


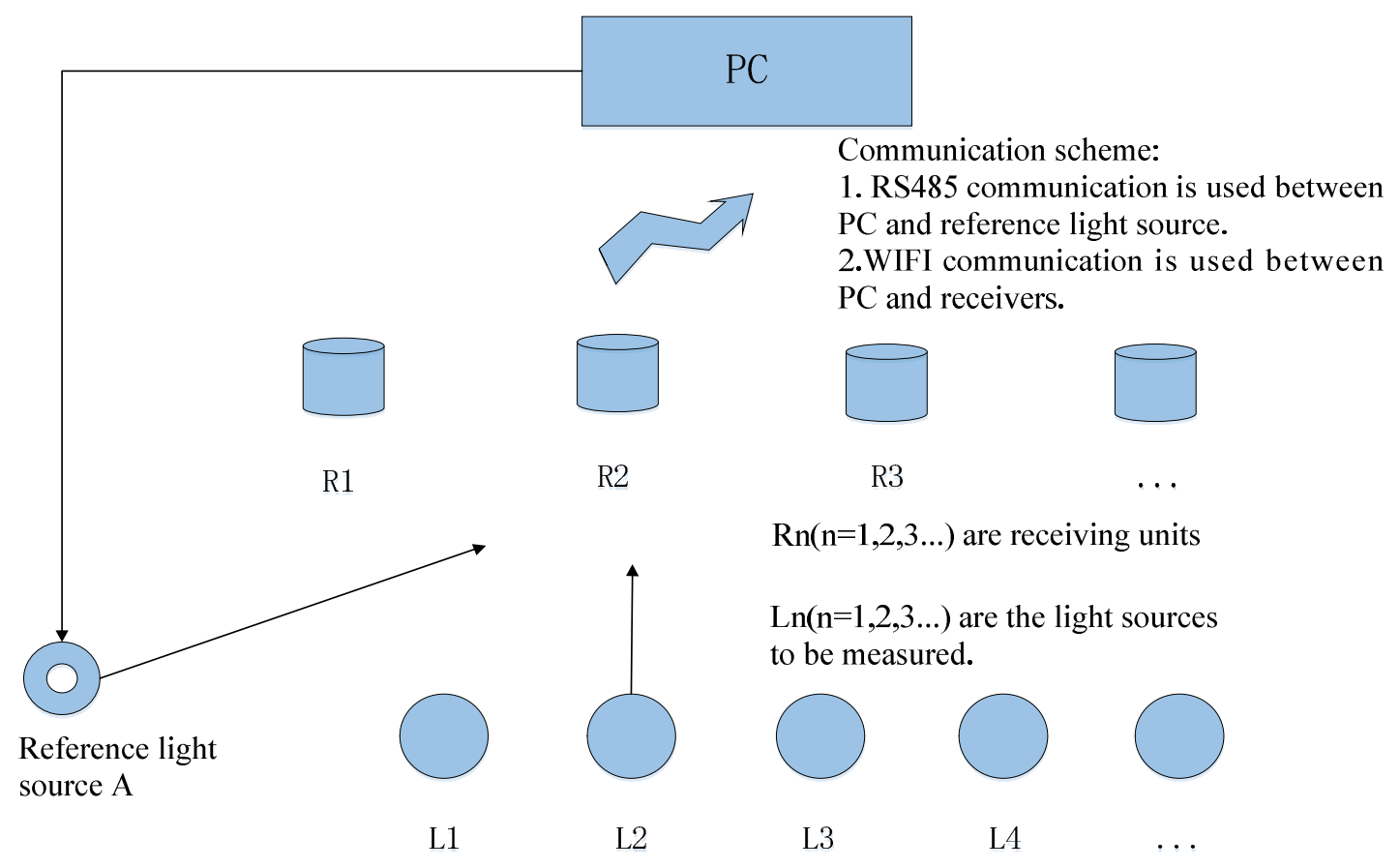

Figure 2. The time division multiplexing System model

The main function of the time-division system is that it can detect the position of multiple points to be tested in space at the same time. Its main work processes:

1. PC sends a start signal to the reference light source A, which starts a round of measurement, and starts the local timer.

2. While the reference light source A receiving a start signal, it has to notify all the light source to be measured to start work within $1 \mathrm{~ms}$.

3. The reference light source A sends a synchronization signal to all the light sources to be measured and sends an optical pulse every $1 \mathrm{~ms}$ immediately after the completion of the synchronization signal transmission for a total of $n$ times (Each corresponding light source to be measured must have a reference light pulses.).

4. After receiving the synchronization signal, all the tested light sources will emit laser pulse after delaying $(\mathrm{m}+0.02) \mathrm{ms}$, where $\mathrm{m}$ is the number corresponding to the serial number of light source to be measured.

5. $\mathrm{R} 1$ to $\mathrm{Rn}$ will receive $\mathrm{n}$ sets of data, which is the time difference between the reference light source and the corresponding light source to be measured.

6. After the time counter reaching a fixed time delay, the PC requests data from all receivers and waits for all receivers to send the measurement results as soon as they receive the request.

The PC solves all positions of L1 to Ln according to the requested measurement result, finishes one cycle of measurement cycle, and turns to first step to prepare the measurement for the next cycle.

\section{Algorithm Description}

For calculating the position of the light source to be measured, it is necessary to integrate the time difference data received by a plurality of receivers to carry out the calculation, and take any one receiving unit 1 to establish the model, which position is set to $\left(x_{a}, y_{a}, z_{a}\right)$, The other receive unit serial number are backward from the letter a and so on, as shown in figure 3 : 


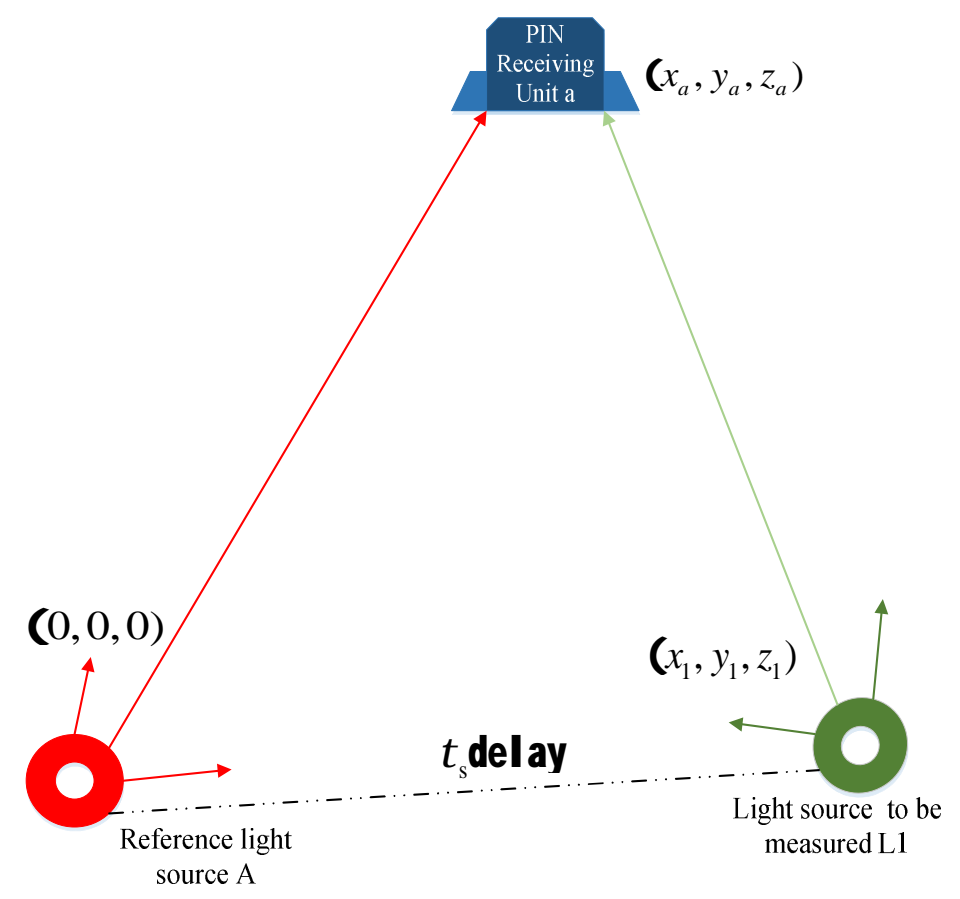

Figure 3. Measurement solution model

From the above model, it is seen that the sum of the distance between the light source L1 to the reference light source $\mathrm{A}$ and the distance between the light source to be measured L1 and the photoelectric receiving unit Rn of the PIN / APD is : $S_{n}=L_{n}+c\left(\tau_{1}-\tau_{s}\right)$, where c is the speed of light. According to the law that the sum of distance between two points to one point is a constant, it can be concluded that the solution set of the L1 position of the light source to be measured is an ellipsoid. The ellipsoid equation is:

$$
\sqrt{x_{1}^{2}+y_{1}^{2}+z_{1}^{2}}+\sqrt{\left(\mathrm{x}_{1}-\mathrm{x}_{\mathrm{a}}\right)^{2}+\left(\mathrm{y}_{1}-\mathrm{y}_{\mathrm{a}}\right)^{2}+\left(\mathrm{z}_{1}-\mathrm{z}_{\mathrm{a}}\right)^{2}}=\sqrt{x_{\mathrm{a}}^{2}+y_{\mathrm{a}}^{2}+z_{\mathrm{a}}^{2}}+\mathrm{c} \cdot\left(\tau_{1}-\tau_{z}\right)
$$

$\left(x_{a}, y_{a}, z_{a}\right)$ is the coordinate of receiver R1, which is known quantity. $\left(x_{1}, y_{1}, z_{1}\right)$ is the coordinate of $\mathrm{L} 1$, which is unknown quantity. $\tau_{1}$ is the time difference given by the R1 receiver, which is known quantity; $\tau_{g}$ is the unknown and non-fixed time difference between the reference light source and the source to be measured; So the solution to four unknown quantities require at least four equations, The $i$ in the equation(3.2) refers to four id of receivers:

$$
\sqrt{x_{1}^{2}+y_{1}^{2}+z_{1}^{2}}+\sqrt{\left(\mathrm{x}_{1}-\mathrm{x}_{\mathrm{i}}\right)^{2}+\left(\mathrm{y}_{1}-\mathrm{y}_{\mathrm{i}}\right)^{2}+\left(\mathrm{z}_{1}-\mathrm{z}_{\mathrm{i}}\right)^{2}}=\sqrt{x_{\mathrm{i}}^{2}+y_{\mathrm{i}}^{2}+z_{i}^{2}}+\mathrm{c} \cdot\left(\tau_{1}-\tau_{z}\right)
$$

Computer the set of equations above is relatively slow, if $1000 \mathrm{~Hz}$ measurement frequency is required and at the same time each measurement cycle time is only less than $1 \mathrm{~ms}$. One cycle contains processes includes many steps, such as: measurement, communication, storage, computing and so on. So the conventional solution equation algorithm can not meet the actual demand, the following two algorithms will be introduced to improve computing efficiency. 


\section{Gauss-Newton Iteration Method ${ }^{[1]}$.}

Gauss Newton iterative method is one of the most important methods in numerical analysis. It is usually used in the solution of differential equations and integral equations, and of course, it is also applicable to the solution of equations or equations.

It is very important to construct iterative functions when using iterative methods to solve nonlinear equations. Newton iteration is one of the commonly used methods to ensure convergence of iterative functions.

This method is used to reduce the number of error solutions and iterations, since the position at this moment should be very close to the previous position, and the iterations can quickly approximate the correct solution. The overall flow chart is shown in Figure 4:

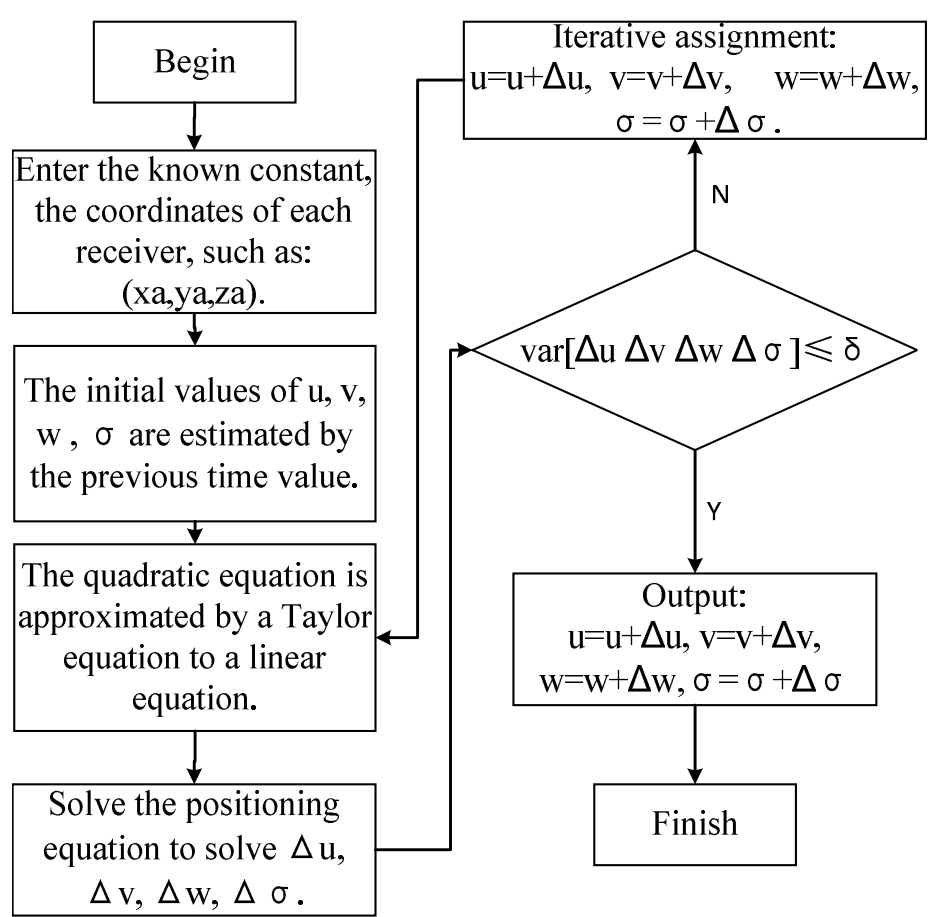

Figure 4. Positioning calculation flow chart

When the number of receivers is more than four, The unknown quantities are: $\left[\begin{array}{ccccc}\Delta u & \Delta v & \Delta w & \Delta \sigma\end{array}\right]$ can be solved through these functions and after the matrix operation, $\left[\begin{array}{llll}u & v & w & \sigma\end{array}\right]=\left[\begin{array}{lllll}u & v & w & \sigma\end{array}\right] \Leftarrow\left[\begin{array}{llll}\Delta u & \Delta v & \Delta w & \Delta \sigma\end{array}\right]$ this iteration is continue after the vector update until the condition of $\operatorname{var}\left[\begin{array}{llll}\Delta u & \Delta v & \Delta w & \Delta \sigma\end{array} \leq \delta\right.$. It is believed that solution of $\left[\begin{array}{llll}u & v & w & \sigma\end{array}\right]$ is sufficiently close to[ $\left[\begin{array}{llll}x_{1} & y_{1} & z_{1} & \tau_{g}\end{array}\right]$. The initial of [ $\left[\begin{array}{llll}u & v & w & \sigma\end{array}\right]$ can be set as $\left[\begin{array}{llll}0 & 0 & 0 & 0\end{array}\right]$ to begin iteration.

\section{Least Square Method ${ }^{[2]}$.}

Through the last chapter above, the nature of the tracking and locating problem can be summed up into the problem of solving nonlinear equations. In the process of actual experiment, we can turn the measurement error into the error of normal distribution. The least square method transforms the localization problem into a nonlinear least squares estimation problem.

It is found that the problem of locating and solving the coordinates can be transformed into a multiple linear regression analysis problem, which can be solved by the least squares method. So we $\operatorname{set}\left[\begin{array}{llll}X_{1 i} & X_{2 i} & X_{3 i} & X_{4 i}\end{array}\right]=X,\left[\begin{array}{llll}x & y & z & S_{d i}\end{array}\right]^{T}=\theta,\left[Y_{i}\right]=Y$. 
Define residual $\mathrm{J}$ of $\theta$ :

$$
I(\theta)=\frac{1}{2} \sum_{i=1}^{m}\left(h_{\theta}\left(x^{(i)}\right)-y^{(i)}\right)^{2}=\frac{1}{2} \operatorname{tr}\left[(X \theta-Y)^{2}(X \theta-Y)\right]
$$

Since the minimum value of the residual is at the extremum:

$$
\frac{\partial J(\theta)}{\partial \theta}=0
$$

Then eventually get:

$$
\theta=\left(X^{T} X\right)^{-1} X^{T} Y
$$

According to the matrix $\left[\begin{array}{lllll}x & y & z & S_{d}\end{array}\right]^{T}=\theta$, the coordinates of the light source to be measured $(\mathrm{x}, \mathrm{y}, \mathrm{z})$ will be obtained.

\section{Kalman filter trajectory optimization}

\section{Application Background.}

In the case of actual detection of the trajectory, even if a given trajectory is designed for the object to be measured, the motion model process will introduce a noise signal due to the influence of various external environments and the random error of the object's own motion. There is an error in the motion itself. But also because of the existence of errors in the measurement instrument itself, which makes the measured object trajectory of the track there will be some deviation.

Kalman filtering can help us to reduce the measurement error of a given trajectory to a large extent, and restore the real three-dimensional space motion trajectory in real-time system.

\section{Introduction to Kalman Filter ${ }^{[3]}$.}

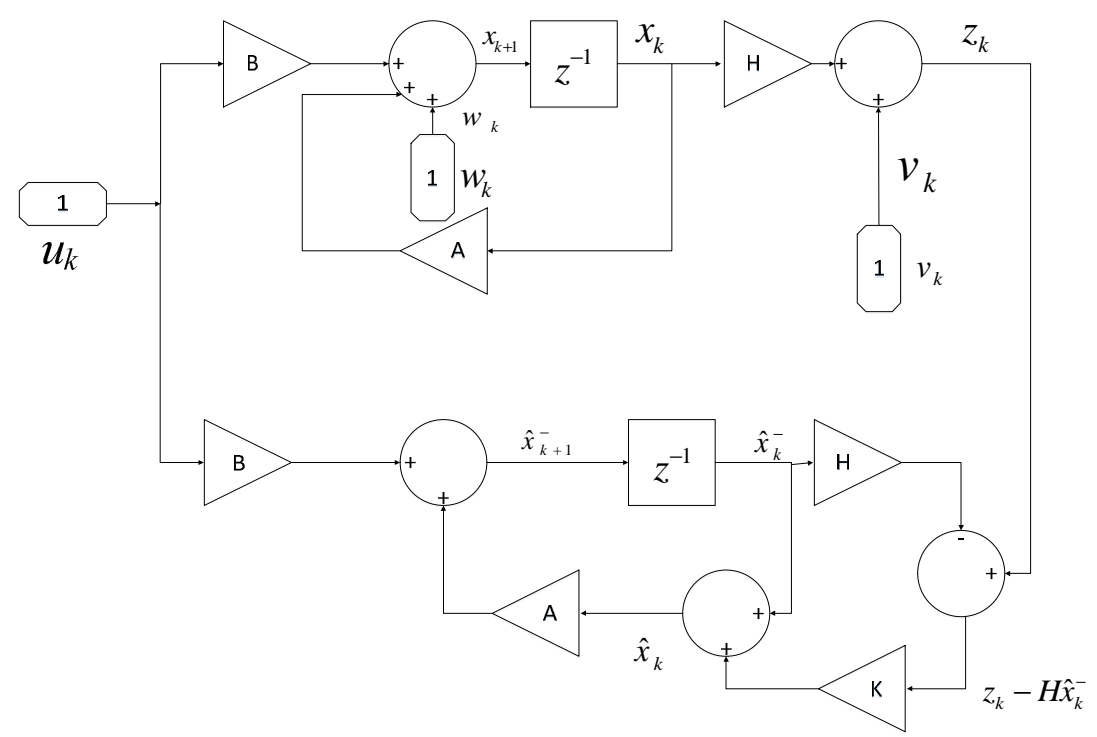

Figure 5. Kalman filter flow chart

The Kalman filter flow chart is shown in Figure 5.

Construct a linear system:

$$
x_{k}=A x_{k-1}+B u_{k-1}
$$

Usually there is a noise term $w_{t-1}$ in the real model, and the variance is $Q_{k}$ :

$$
x_{k}=A x_{k-1}+B u_{k-1}+w_{k-1}
$$

The relationship between measurable quantity $\mathrm{z}$ and state of the amount $x_{k}$ :

$$
z_{k}=h x_{k}+v_{k}
$$


$v_{k}$ is the error in the measurement process, and it follows the Gaussian distribution and the variance $\mathrm{R}_{\mathrm{k}}$ is used to estimate the system:

Let the state to be $\mathrm{k}$ at this moment, according to the model of the system, the state $\mathrm{k}$ can be predicted based on the previous state $\mathrm{k}-1$ of the system:

$$
\hat{x}_{k}^{-}=A \hat{x}_{k-1}^{-}+B u_{k-1}
$$

Calculate the error covariance matrix between the predicted and true values:

$$
\boldsymbol{P}_{k}^{-}=A P_{k-1} A^{T}+Q_{k}
$$

According to $P_{k}^{-}$can be obtained in the k state of the Kalman filter gain matrix K:

$$
K_{k}=P_{k}^{-} H^{T}\left(H P_{k}^{-} H^{T}+R_{k}\right)^{-1}
$$

\section{Filter Equation.}

Based on the series of Kalman filter mathematic models given above, the system state vector is chosen $\mathrm{X}=\left[\begin{array}{llllll}\mathrm{x} & v_{x} & y & v_{y} & z & v_{z}\end{array}\right]$, acceleration $\left[\begin{array}{lll}a_{x} & a_{y} & a_{z}\end{array}\right]$ is set as a controlled variable, from this we can get the system equation as follows:

$$
X_{k}=A \times X_{k-1}+B \times U+W_{k-1}
$$

In the above equation:

$$
A=\left[\begin{array}{cccccc}
1 & \Delta t & 0 & 0 & 0 & 0 \\
0 & 1 & 0 & 0 & 0 & 0 \\
0 & 0 & 1 & \Delta t & 0 & 0 \\
0 & 0 & 0 & 1 & 0 & 0 \\
0 & 0 & 0 & 0 & 1 & \Delta t \\
0 & 0 & 0 & 0 & 0 & 1
\end{array}\right] \quad B=\left[\begin{array}{cccc}
\frac{1}{2} \Delta t^{2} & 0 & 0 \\
\Delta t & & 0 & 0 \\
0 & \frac{1}{2} \Delta t^{2} & 0 \\
0 & \Delta t & & \frac{1}{2} \Delta t^{2} \\
0 & 0 & \Delta t
\end{array}\right]
$$

$W_{k-1}$ is the noise vector of system;

Taking into account the measurement error:

$$
Z_{k}=H_{k} X_{k}+V_{k}
$$

In the (4.8) equation: $H=\left[\begin{array}{llllll}1 & 0 & 0 & 0 & 0 & 0 \\ 0 & 0 & 1 & 0 & 0 & 0 \\ 0 & 0 & 0 & 0 & 1 & 0\end{array}\right] ; \boldsymbol{V}(k)$ is the measurement noisy sequence.

The system noise and measurement noise are zero-mean Gaussian white noise sequences. From the above we can see that the value of the filter gain matrix $K_{k}$ is mainly determined by the system noise variance $Q_{k}$ and the measured noise variance $R_{k}$. It can be seen that whether the system noise variance $Q_{k}$ and the measurement noise variance $R_{k}$ are appropriate affects the quality of the filtering result directly. Therefore, we choose adaptive filtering to overcome this shortcoming of traditional filtering.

Adaptive filtering is the use of measurement data. Filter will determine constantly whether the dynamic target changes by itself in the recursion. When it is judged that there is a change, then further decide whether to regard the change as stochastic interference to the noise, or to modify the original dynamic model to adapt to the dynamic target. Another purpose of adaptive filtering is to continuously estimate and correct the dynamic noise variance $\mathbf{Q}_{k}$ and the measured noise variance $R_{k}$ by the filter itself when they are unknown or uncertain. In this paper, we choose a modified Sage-Husa filter ${ }^{[4]}$ (maximal posteriori estimator):

$$
\begin{aligned}
& \varepsilon_{k}=Z_{k}-H_{k} \hat{X}_{k}^{-} \\
& \hat{R}_{k+1}=\left(1-d_{k-1}\right) R_{k}+d_{k}\left(\left[I-H_{k} K_{k}\right] e_{k} \varepsilon_{k}^{T}\left[I-H_{k} K_{k}\right]^{T}+H_{k} P_{k} H_{k}^{T}\right) \\
& Q_{k+1}=K_{k} \varepsilon_{k} \varepsilon_{k}^{T} s K_{k}^{T}+P_{k}-A P_{k-1} A^{T}
\end{aligned}
$$


Where $d_{k}$ is the weighted index, $d_{k}=(1-b)+\left(1-b^{k+1}\right), 0<\mathrm{b}<1, \mathrm{~b}$ is called the forgetting factor $^{[5]}$.According to the actual situation to make appropriate adjustments, the general value of the range of 0.95 to $0.99 . \varepsilon_{k}$ becomes the innovation of $\mathrm{k}$ state, which is used to represent the difference between observed and predicted observations.

The improved Sage-Husa adaptive algorithm not only reduces the complexity of the conventional Kalman algorithm, but also realizes the real-time and adaptive filtering of the system during operation, thus improving the accuracy of the system fusion.

\section{Simulation Analysis.}

The simulation conditions are the same as above. The forgetting factor is 0.98 , and the simulation experiment simulates the three modes of points' motion to be measured: uniform motion, uniform acceleration motion and helix trajectory movement.

The simulation system noise variance is: $Q_{k}=\left[\begin{array}{ccc}0.01 & 0 & 0 \\ 0 & 0.01 & 0 \\ 0 & 0 & 0.01\end{array}\right]$.

The measured noise is determined by the actual receiver module to fit the measured results shown in Figure 6:

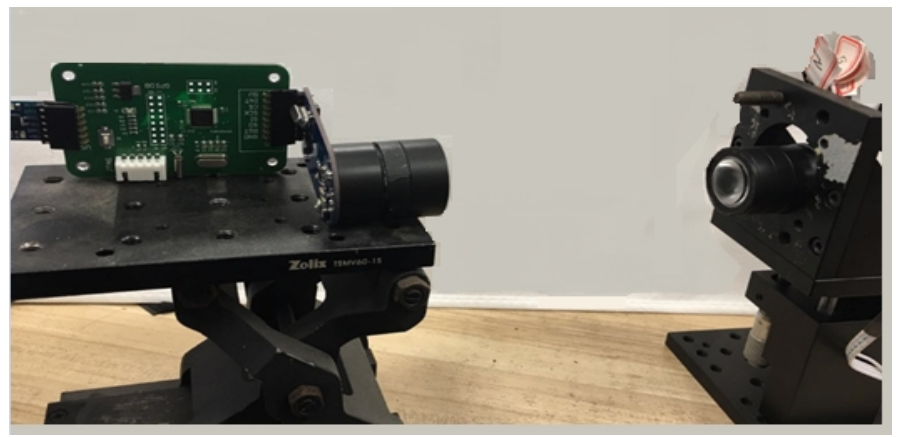

Figure 6. Receiver module accuracy measurement experiment

In the experiment, the left-side receiving module measure the time difference between the two rising edge of the light pulse $\mathrm{T}$.

Convert measurement time to distance: $s=c \cdot T$. The data of 500 groups collected by the two methods were analyzed statistically. The first kind is PIN photodiode to receive data. The fitting function is:

$$
\text { Normalfit }(S)=a \times e^{-\left(\frac{s-b}{a}\right)^{2}}
$$

The APD photodiode to receive data fitting function is:

\begin{tabular}{|c|c|c|c|c|}
\hline $\mathrm{a}$ & $\mathrm{b}$ & $\mathrm{c}$ & R-square & MSE \\
\hline 41.51 & 0.7478 & 0.01249 & 0.9818 & 0.0103 \\
\hline
\end{tabular}

Table 1. The coefficient of fitting function from APD

The APD measurement data is subject to Gaussian distribution $N\left(\mu, \sigma^{2}\right), \mu=0.7478, \sigma=0.0103$. The coefficient of APD is shown in Table 1. In order to get close to the real environment, the measurement noise is introduced that determined by the actual measurement process.

The simulation results of the adaptive filtering algorithm are shown as follows: 


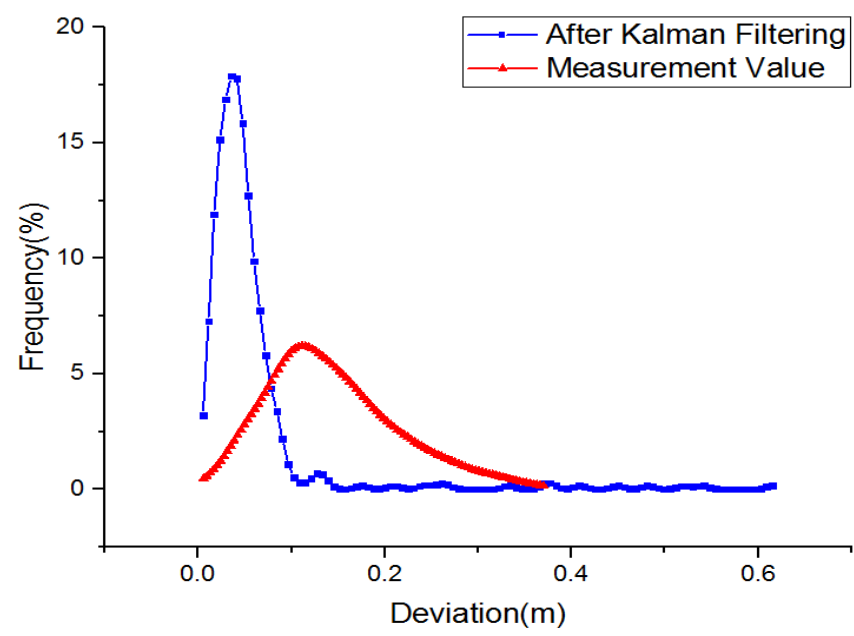

Figure 7. Deviation of uniform motion filtering

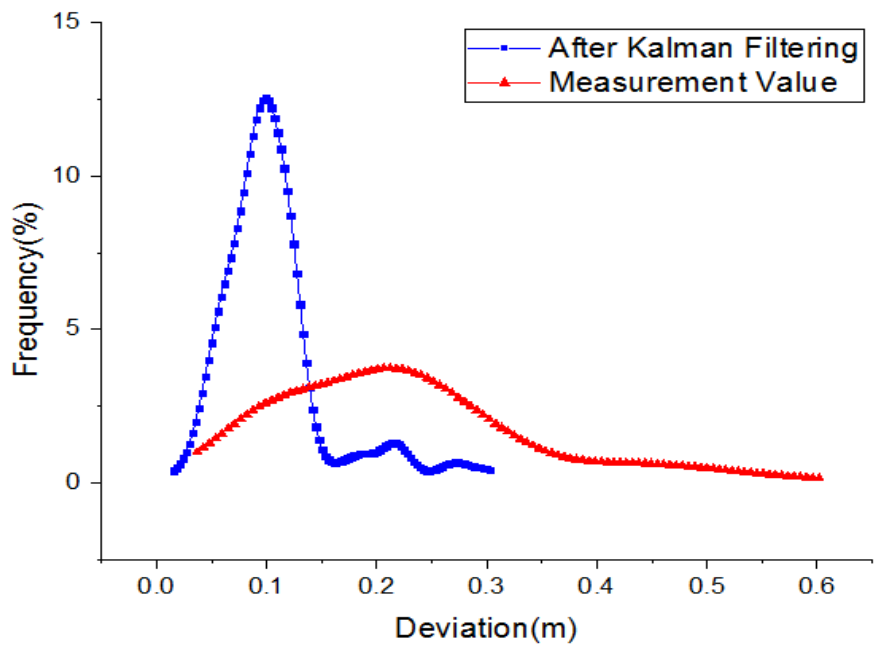

Figure 8. Deviation of uniform acceleration motion filtering

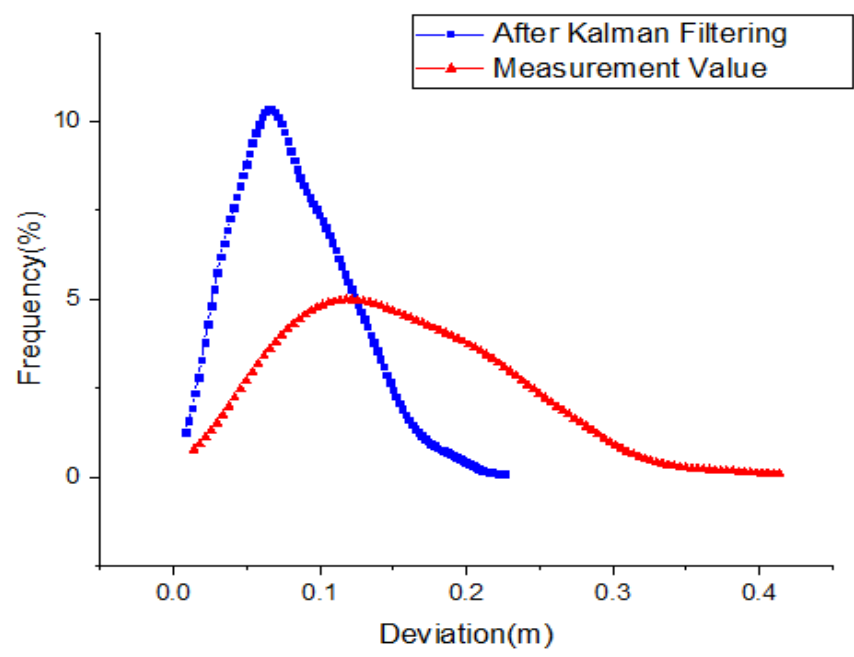

Figure 9. Helical trajectory motion 
In the above three graphs, the blue line is the probability density distribution map of the deviation between the position value from the true motion and the after the optimal estimate after Kalman filter; where the deviation is defined as the absolute distance from the optimal estimated position to the true track position. The red line is the probability density distribution of the deviation between actual measured value and the true motion trajectory. The deviation is defined as the absolute distance from the actual measurement position to the true track position.

From these three states, it can be seen that the probability density distribution of the blue curve is shifted to the left of the red line and the blue peak of the probability density curve is higher. The result shows that the accuracy of the whole data is improved greatly after Kalman filter.

The figure $\backslash$ shows the overall error level decreased $48.26 \%$. The figure 8 shows overall error level decreased $60.29 \%$. The figure 9 shows overall error level decreased $49.49 \%$.

This result proved that the Kalman filter is used in dynamic positioning real-time tracking can be a good way to reduce the error from system itself, and measurement error. Sage-Husa improved method can effectively restore the actual position of the object and the true trajectory.

\section{Conclusions}

I This paper mainly introduces the advantages and disadvantages of the current indoor positioning technology, and puts forward a new indoor positioning technology according to different needs, and introduces the positioning system in detail. The main features of the system include:

I Distributed sensor system, it can provide more accurate position information of higher refresh rate. A large number of information of sensor gives the developer the possibility of using the algorithm to optimize the accuracy of the system, so that the spatial positioning system does not exist physical limitations.

I The use of reference light source for sensor synchronization is proposed, which solves the core problem of technology of TDOF measurement which requires high synchronization of the receiver.

Besides, in this paper, two kinds of location algorithms, Gauss Newton iterative algorithm and least squares estimation method, which are commonly used in GPS-like positioning methods, are introduced in detail and applied in this paper. And the error of the measurement of the detector simulation is obtained by experiment.

After the spatial position is calculated by the position solution algorithm, the sources of the error of the dynamic position measurement in space are discussed. The improved Sage-Husa adaptive algorithm of the Kalman filter is introduced and the filtering equation is listed. The algorithm reduces the complexity of the conventional Kalman filter. Finally, the effect of Kalman filter is verified by simulation of three motion models.

\section{Acknowledgements}

I would like to thank my colleagues for innumerable contributions to the discussion about embedded development and mobile applications. I would like to thank Zhigang Tao for his help in hardware development and network architecture specifically. And I want to thank my tutor's guide and his financial support. He always provided many useful comments on the text and whole project.

\section{References}

[1] Feng Du. GPS/BDS composite system of single point location based on Gauss-Newton iteration method[J]. Science and Technology, 2016,7:129. (in Chinese)

[2] Xiaochang Cheng, Shaoying Su, Yueke Wang, et al. Few Location Algorithms Comparison in Quasi-GPS Ultrasonic Location System[J]. Chinese Journal of Sensors and Actuators, 2007,20(3): 698-702. (in Chinese)

[3] Wenyin Gong. Research on Evolutionary Kalman Filter and Its Application[D]. Wuhan: China University of Geosciences, 2007. (in Chinese)

[4] lijia Xu, Yangzhou Chen, Cui Ping-yuan. Fuzzy Information Fusion in Integrated Navigation System[J]. Journal of System Simulation, 2005, 17(1): 214-128. (in Chinese) 
[5] Jiayuan Hu, Zhengdong Sha. Adaptive beamforming using Kalman filter with forget factor[J]. Chinese Journal of Radio Science, 1999, 14(2): 148-152. (in Chinese)

[6] Shouxin Zhang, People's Liberation Army General Armament Department. GPS Technology and Application [M]. Beijing: National Defense Industry Press, 2004. 